We try to publish authors' responses in the same edition with readers' comments. Time constraints might prevent this in some cases. The problem is compounded in a bimonthly journal where continuity of comment and redress are difficult to achieve. When the redress appears 2 months after the comment, 4 months will have passed since the article was published. Therefore, we would suggest to our readers that their correspondence about published papers be submitted as soon as possible after the article appears.

\section{Teaching Information Mastery}

To the Editor: Slawson and Shaughnessy, in their article in the November-Decemer 1999 issue of The Fournal, ${ }^{1}$ described the results of an innovative 2-year longitudinal intervention to teach family practice residents the techniques and philosophy of evidence-based medicine and information mastery. Their results showed a $17 \%$ more positive attitude toward use of the literature, an $8 \%$ difference in self-perceived ability to evaluate clinical trials, and a $9 \%$ increase in the self-reported frequency of use of information sources. These are important intermediate findings in our quest for proof that the teaching of evidence-based medicine makes a difference to the current behavior and furure practice of clinicians.

The authors, however, failed to acknowledge any inherent weaknesses of this study. This study involved only 29 residents in two programs. Because there was also no control group (which would have excluded this study from the review conducted in 1998 by Norman and Shannon ${ }^{2}$ ), it is impossible to know how much of this change would have occurred naturally, without the educational intervention.

Although the instrument was well validated, the selfreported constructs were subjective rather than objective; thus, it is difficult to translate their meaning into measurable behavior change. It is also difficult to judge the clinical significance of the small (but statistically significant) changes from their preintervention to postintervention scores.

We do not wish to attack the authors in any way. In fact, we hold them in considerable personal esteem, all of us having attended their excellent course on information mastery at the University of Virginia. Like Slawson and Shaughnessy, we are struggling in our attempts to show that our interventions change learner behavior in a clinically important way. Given the modest evidence of their program's effectiveness, and the methodologic weakness of its evaluation, we were especially surprised by the strength and scope of their conclusions: "Offering a structured curriculum to family practice residents creates dynamic, confident, and independent clinicians skilled in the art of information mastery." Increased dynamism, confidence, and independence appear to lie beyond what could realistically be inferred from the study.
Slawson and Shaughnessy have been leaders in developing the concept and techniques of teaching information mastery, but proponents of evidence-based medicine-and we are card-carrying members of that groupshould not allow their enthusiasm to override their critical appraisal skills. Perhaps Slawson and Shaughnessy have taught us too well.

Alison E. Dobbie, $\mathrm{MD}, \mathrm{ChB}$

F. David Schneider, MD, MSPH

Robert Ferrer, MD

University of Texas Health Science Center

San Antonio

\section{References}

1. Slawson DC, Shaughnessy AF. Teaching information mastery: creating informed consumers of medical information. J Am Board Fam Pract 1999;12:444-9.

2. Norman GR, Shannon SE. Effectiveness of instruction in critical appraisal (evidence-based medicine) skills: a critical appraisal. CMAJ 1998;158:177-81.

The above letter was referred to the authors of the article in question, who offer the following reply.

To the Editor: We appreciate the attention of Drs. Dobie, Schneider, and Ferrer regarding our article on teaching information mastery. We agree with their critique of our work to date. Our main thrust in publishing this article was not to evaluate our curriculum rigidly, but instead to get it down on paper so that others would have more direct access to it. We were encouraged by this preliminary evaluation of its usefulness. We have focused our academic efforts on the consumer education division of the information business as outlined in the article (we appreciate the positive feedback on our workshops). We hope publication of this work will encourage others to complete the information business cycle by constructively evaluating our work. Evaluation of one's own "children" is usually best done by others.

David C. Slawson, MD Charlottesville, $\mathrm{Va}$ Allen F. Shaughnessy, PharmD Philadelphia, $\mathrm{Pa}$

Congestive Heart Failure Clinical Outcomes Study in a Private Community Medical Group

To the Editor: The article by Civitarese and DeGregorio ${ }^{1}$ on congestive heart failure clinical outcomes is an important contribution as a descriptive study on implementing a disease management program in private practice. Though supportive of their process, we are suspicious of their conclusions.

In presenting the data, it is unclear when the actual intervention took place. Was it throughout the data collection period? Are there comparison data from before 
the intervention or a second comparison group? Are outcomes really improved? One outcome is mortality, and a quick glance at the table suggests that in-hospital mortality is increasing with time. Logistic regression analysis is reported in the Results section, though no mention is made in the Methods of why or how this was done. No denominator for the number of heart failure patients in the practice is reported. Although the authors suggest that a reduced number of admissions resulted from the process, the use of angiotensin-converting enzyme inhibitors in the outpatient settings did not appear to change, as evidenced by its constant rate of use (or nonuse) among those admitted with heart failure. Additionally, data sets such as those used by insurance companies do not classify heart failure based on left ventricular ejection fraction measurement. ${ }^{2,3}$ As written, the article serves as an excellent guide to implementing an excellent quality improvement intervention. The lack of a comparison group and the insufficient data available to examine rates of hospital admissions for heart failure prevent us from accepting the conclusions of reduced hospitalizations at this time. Even though the authors' assertions might ultimately prove to be valid, we would encourage more caution in the stating of conclusions.

Paul A. James, MD

Laurene Tumiel, MA

State University of New York Buffalo

\section{References}

1. Civitarese LA, DeGregorio N. Congestive heart failure clinical outcomes study in a private community medical group. J Am Board Fam Pract 1999;12:467-72.

2. Aetna US Healthcare data. PCP cardiac performance report. 5/1/96-4/30/97 and 4/1/97-3/31/97. Pittsburgh: Aetna US Healthcare, 1997. (Report reflecting sampling of PCP patients evaluated for congestive heart failure and appropriate angiotensin-converting enzyme inhibitor therapy.)

3. Croft JB, Giles WH, Roegner RH, Anda RF, Casper ML, Livengood JR. Pharmacologic management of heart failure among older adults by office-based physicians in the United States. J Fam Pract 1997;44:382-90.

The above letter was referred to the authors of the article in question, who offer the following reply.

To the Editor: As discussed in the Study Design and Practice Guideline sections of the article, the guideline was introduced at the outset of the study period and revisited each month at our regularly scheduled continuing medical education meetings. Also, as mentioned, the physicians were apprised of their performance data at quarterly quality improvement meetings; therefore, the intervention indeed occurred throughout the study period. As mentioned in the Conclusions section of the article, we believed this was paramount to our success.

We did not measure our performance at any time before the intervention. There was no control population in our study. Our intent was to measure whether the guideline would improve our care for congestive heart failure. It was not our intent to compare our performance to that of another medical group. We believed it would be impossible or unethical to develop a control population of patients within our medical group.

As stated in the Conclusions section of our paper, reducing hospital admissions for systolic congestive heart failure has been a valid outcome measure in a previously published landmark trial. We therefore conclude that outcomes improved throughout our study. Statistical regression was the simplest modeling tool to support our findings. The study was not powered to develop any statistical significance in regard to mortality; therefore, we would reserve judgment relating to any mortality statistics presented.

Because this population was not a closed population, there is no fixed denominator. The statistical relevance of the data, however, lies in the five consecutive quarters that we experienced progressively lower numbers of admissions for systolic dysfunction while recording remarkably steady numbers of admissions for diastolic dysfunction. The only way in which these data could be considered faulty would be if only our systolic congestive heart failure patients somehow self-directed their care to other hospitals. We consider that extremely unlikely.

Selecting only those patients who required admission to the hospital for congestive heart failure as a fair representation of angiotensin-converting enzyme (ACE) inhibitor use within our entire outpatient congestive heart failure practice is in error. In fact, one could intuitively expect that the subset of patients requiring admission would likely have the lowest rates of ACE inhibitor use.

Finally, as stated in the conclusion, we would have preferred to have completed our own measurement of ACE inhibitor use by our physicians in the outpatient setting. The group believed, however, that the additional demands required to complete the audit exceeded our financial and human resources. As a best alternative, Aetna US Healthcare data were used as surrogate data. Though we agree that it is possible, we consider it highly unlikely that the rise in ACE inhibitor use as measured by Aetna US Healthcare was the result of increased use primarily in patients with diastolic dysfunction.

Louis A. Civitarese, DO

Nicholas DeGregorio, MD

Preferred Primary Care Physicians Pittsburgh

\section{Prenatal Testing and Counseling for Down Syndrome}

To the Editor: This letter is in response to the article entitled "Multiple Marker Screening for Down Syndrome-Whom Should We Screen?" by Dr. Sara Cate and Susie Ball (7 Am Board Fam Pract 1999;12:367-74). An otherwise clear and concise review of prenatal genetic screening was marred by some muddled statements that, I suppose, were meant to reflect ethical issues.

The authors noted that family physicians and internists were more likely than other specialists to interject their own opinions regarding abortion. Male physicians were noted to be more likely than female physicians to 